Cahiers d'études italiennes

\title{
Carlo Sgorlon: Ideologia e guerra
}

\section{Stefano Magni}

\section{OpenEdition}

\section{Journals}

Edizione digitale

URL: http://journals.openedition.org/cei/292

DOI: $10.4000 /$ cei.292

ISSN: 2260-779X

\section{Editore}

UGA Éditions/Université Grenoble Alpes

\section{Edizione cartacea}

Data di pubblicazione: 15 juin 2005

Paginazione: 235-251

ISBN: 978-2-84310-066-6

ISSN: $1770-9571$

Notizia bibliografica digitale

Stefano Magni, «Carlo Sgorlon: Ideologia e guerra», Cahiers d'études italiennes [Online], 3 | 2005, online dal 15 décembre 2006, consultato il 27 mars 2021. URL: http://journals.openedition.org/cei/292 ; DOI: https://doi.org/10.4000/cei.292 


\title{
CARLO SGORLON \\ IDEOLOGIA E GUERRA *
}

\author{
Stefano Magni \\ Université Stendhal - Grenoble 3
}

Carlo Sgorlon ha pubblicato tre romanzi ambientati nel Friuli della Seconda Guerra mondiale : L'armata dei fiumi perduti (1983), La foiba grande (1991), La malga di Sîr (1995).

Con quest'analisi intendo indagare la posizione dell'autore di fronte alle convenzioni che abitualmente reggono il genere del romanzo storico. Per farlo mi confronterò anche con i giudizi più consolidati della critica. Claudio Toscani, infatti, in Invito alla lettura di Sgorlon, parla del candore epico-religioso con cui l'autore guarda le cose ; di una concezione della vita secondo i sentimenti dell'amore, della pietà, della comprensione, della donazione e della consolazione. Per Bruno Maier Sgorlon è il cantore o l'aedo di un piccolo mondo contadino. Liana Nissim parla di profonda compassione per l'umanità, di simpatia per le vittime della storia. Dal confronto con le convenzioni del genere possono nascere nuove ipotesi interpretative e nuove prospettive critiche?

Presento, per cominciare, alcuni fatti storici a cui fa riferimento la materia di tutti e tre i libri : il 10 sett. 1943, quindi solo due giorni dopo l'8 settembre 1943, prima ancora che nasca la Repubblica di Salò, il Friuli è annesso direttamente al Terzo Reich nella provincia dell'Adriatisches Küstenland. A Trieste viene inviato uno staff efficientissimo, proveniente dalla Polonia. Ciò dimostra l'importanza strategica della regione per i tedeschi ${ }^{1}$. Subito nasce anche la resistenza con le prime brigate Garibaldi

1. Cfr. Dallo squadrismo fascista alle stragi della risiera: Trieste-Istria-Friuli 1919-1945, Trieste, Aned, 1974 , p. 90 e p. 96 : «In data 10 settembre ' 43 cioè ad appena due giorni dall'armistizio fra l'Italia e gli angloamericani (il che confermava come il piano fosse stato predisposto da tempo) Hitler [...] stabiliva la creazione delle due zone di operazioni del « Litorale " e delle " Prealpi " (la prima formata dalle 
d'Italia. Quindi si costituisce la Repubblica di Salò e con essa arriva la X mas. Ad Est c’è la Jugoslavia con i partigiani titini, comunisti. Nelle montagne della Carnia, alla fine del luglio 1944 le formazioni partigiane delle Divisioni Garibaldi (comuniste) si rendono padrone di un territorio vasto quanto il Lussemburgo e popolato da più di ottantamila abitanti, la zona libera più grande d'Italia.

Per combattere questo piccolo «Stato » partigiano, protetto dai monti e difficile da attaccare, il Reich decide di insediare nei paesi friulani i cosacchi, il popolo della steppa che non si è mai piegato al comunismo e che in patria è perseguitato da Stalin. In cambio dell'operazione militare, i nazisti promettono ai cosacchi la regione intera come futura patria.

L'Armata dei fiumi perduti ${ }^{2}$ prende l'avvio da queste vicende, ma nel romanzo si dà pochissimo peso all'avvenimento, che pure fu la ragione per cui i cosacchi invasero il Friuli. L'esistenza della Zona libera è menzionata nel romanzo solo una volta, in un contesto marginale, senza nessun dettaglio : Ugo, un giovane che fa la spola tra i partigiani e il villaggio, dice che i tedeschi non avrebbero mai osato entrare, appunto, nella Repubblica di Carnia (ibid., p. 38). Il dato ci assicura, perlomeno, che la storia si svolge nella zona libera : mancando altri riferimenti concreti, sarebbe potuto sorgere un legittimo dubbio.

Nel romanzo, infatti, solo in modo molto vago Marta, che rappresenta il punto di focalizzazione più frequente dell'intera vicenda e uno dei personaggi centrali della storia, comincia a sentir parlare della formazione di bande partigiane in montagna, estranee alla vita popolare. Gli abitanti dei villaggi conoscono questa realtà sommersa, come si trattasse di un misterioso oggetto lontano e segreto.

La Repubblica della Carnia fu una democrazia con elezioni aperte anche alle donne, in cui furono aperte scuole e organizzate assemblee popolari che, stando ai documenti, erano veramente popolari e capillari. Ma di tutta quest'esperienza non v'è traccia nel romanzo. Vediamo inoltre com’è descritto in modo pacifico e discreto l'arrivo dei cosacchi : «presero possesso delle stanze semivuote, o riempite al massimo di attrezzi, di fagioli, di grano, di mais. Portarono dai fienili grandi bracciate di paglia e dissero : "Kazak dormire qui"» (ibid., p. 48). La scena è dolcissima, ma

province di Udine, Gorizia, Trieste, Pola, Fiume, Lubiana, la seconda da quelle di Trento, Bolzano, Belluno) [...] L'operato di Globocnick in Polonia fino al '43, quando venne trasferito a Trieste, va tenuto presente $[\ldots]$ per la comprensione dei fini politico-terroristici che motivavano il suo trasferimento in una zona nevralgica e di forte attività partigiana come il « Litorale ». [...] a Trieste sarebbe stato trasferito l'intero «staff » dell'Aktion Reinhard. »

2. Carlo Sgorlon, L'armata dei fiumi perduti, Milano, Mondadori, 1983. 
nessuna documentazione storiografica la supporta. Nel 1944 i cosacchi conquistarono la Carnia in due mesi di battaglie che posero termine all'esperienza della zona libera, e poi occuparono, da padroni, le case degli autoctoni. Più di trecento partigiani morirono, molti civili furono uccisi $\mathrm{o}$ deportati, innumerevoli furono i casi di violenza ; ci furono interi villaggi saccheggiati, fienili incendiati ; non mancarono le razzie di animali da stalla e da cortile. La documentazione storiografica è abbondante.

In una nota nel paratesto Sgorlon precisa, inserendosi così in una tradizione letteraria ben precisa, che il romanzo è un « misto di storia e d'invenzione ». Aggiunge poi che l'impostazione generale del racconto è costruita " sopra i miei ricordi e la pietà per quello strano popolo di invasori primitivi $[\ldots .$. ». Sgorlon è nato a Cassacco (poco a Nord di Udine) nel 1930. Qual è stata la sua esperienza dell'invasione cosacca ? Si tratta di una conoscenza diretta delle battaglie della Carnia, o piuttosto di un'eco lontana percepita dalle retrovie ? In ogni caso il suo atteggiamento così deferente non trova riscontro in nessuna documentazione. Inoltre, il giovane cosacco Ghirei, che focalizza spesso su di sé la visione di tutto il suo popolo, è dipinto quasi come un novello Giovanni Drogo di fronte al deserto dei Tartari, con il nemico invisibile e una battaglia che non arriva mai. Storicamente gli scontri ci furono, non solo, ma Marco Cesselli sottolinea che nel corso della battaglia di Nimis "si distinguevano nei massacri i cosacchi del generale Krasnov, le cui angherie, ruberie e atrocità arrivarono al punto da preoccupare gli stessi fascisti, che fecero timidi passi presso il Comando germanico ${ }^{3}$ ".

Il punto di vista della narrazione, poi, è spesso quello corale del popolo cosacco che vede i partizany come nemici oscuri calati in un anonimato distante. Le pallottole della resistenza sono «senza scopo, senza direzione, senza senso, sparate da qualche incosciente soltanto perché in un certo momento di euforia o di rabbia si trovava un'arma tra le mani ${ }^{4}$ ". La guerra dei boschi è descritta come un'assenza. I partigiani sono continuamente definiti come invisibili, inafferrabili, i luoghi che vivono sono inviolabili e leggendari e di loro si conoscono solo vagamente i favolosi nomi di battaglia, imprendibili anche loro : il principale è Vento.

Perché i partigiani sono definiti come esseri senza corpo ? Si potrebbe pensare che ciò sia dovuto ad un'immediata identificazione tra il soldato dei boschi e l'ignoto, che è la sua principale arma. Ma un dubbio ci induce a approfondire l'analisi. I partigiani paiono esclusi dal « corpo » sociale, nel

3. Marco Cesselli, Porzûs due volti della resistenza, Milano, La Pietra, 1975, p. 13.

4. Carlo Sgorlon, op. cit., p. 58. 
quale sono riassorbiti nel momento in cui perdono il loro ruolo di armati e tornano ad essere contadini e lavoratori, secondo l'ordine delle cose. C'è una scissione tra la persona civile e il suo essere partigiano. Così, verso la fine, si scopre il volto del famigerato Vento : « $\operatorname{Era~Ivos~}^{5}$ [...] il famoso capo partigiano, che finita la guerra era tornato nel paese di Marta. Non voleva a nessun patto essere chiamato Vento, né per solito accettava il discorso sulla guerra e sulla lotta partigiana ${ }^{6}$ ". Il volto del resistente appare solo a scontro terminato, in un certo senso solo dopo un'abiura della propria esperienza, e quando ormai non accetta più il suo nome di battaglia. È il momento in cui avviene l'integrazione del soggetto nel tessuto fisico e morale della collettività. C'è quasi una leggera ironia nella peripezia finale di Vento e Ghirei, opposti durante la guerra, ma subito amici in tempo di pace : "Vento e Ghirei andavano a castagne e ritornavano con lo zaino stracarico " (ibid., p. 290).

$\mathrm{Al}$ corpo indefinito, ineffabile, del partigiano si oppone invece quello glorioso del cosacco, visto non con gli occhi dell'italiano occupato, ma con quelli epici della letteratura russa che ne ha osannato le doti di cavaliere e di guerriero. Il cosacco è atletico, danza e combatte, ma in ciò si riflette l'irrealtà della fonte letteraria, del mito che Sgorlon, scrittore di miti, raccoglie da altri testi. Nei due estremi di assenza fisica da un lato e presenza gagliarda dall'altro, si legge in ogni caso la mancanza di realtà sanguigna, si riconosce la convenzione letteraria della finzione.

Nel romanzo c'è sicuramente molta pietà per i cosacchi - che ebbero un destino assolutamente tragico - ma questo sentimento è trasmesso al lettore grazie anche ad alcune omissioni storiche e a discapito della lotta partigiana.

Il finale, che si concentra sulla partenza dolorosa delle famiglie cosacche, tace i durissimi scontri degli ultimi giorni di guerra di cui i cosacchi furono tenaci protagonisti. I guerrieri del Don, infatti, opposero un'ultima strenua resistenza, anche quando gli stessi tedeschi già desistevano. Oltre ad alcuni scontri militari, vanno ricordati i lugubri strascichi delle battaglie : per esempio, alcuni capi partigiani scesero a concordare le modalità della ritirata e furono uccisi. Per vendetta i resistenti giustiziarono i prigionieri cosacchi e attaccarono la colonna in marcia. Come ultima vendetta da parte cosacca, ci fu la distruzione di un intero paese della Carnia sulla strada dell'esodo. Di questi avvenimenti non c’è traccia

5. Che il nome alluda ad origini slave o forse anche sovietiche ? Che il partigiano, ancora per esclusione, non sia un vero friulano?

6. Carlo Sgorlon, op. cit., p. 289. 
nel romanzo. Eppure, le fonti storiografiche a cui lo scrittore dichiara di essersi appoggiato sono ben più dettagliate. Invece, in un romanzo come La terra impossibile di Bruna Sibille-Sizia ${ }^{7}$ la patina della finzione letteraria è esigua, e si ha una descrizione decisamente più concreta e carnale di fatti e persone ${ }^{8}$.

Le regole del romanzo storico - come tracciate in modo abbastanza unanime da una teoria che si è sviluppata già abbondante dal XIX secolo - consistono nel riferire gli avvenimenti della finzione a fatti storiograficamente riscontrabili e nel creare personaggi inventati che agiscono all'interno di questa cornice.

L'armata dei fiumi perduti, dunque, non contravviene a questo sistema di prescrizioni, semplicemente, in modo legittimo, il romanzo omette molti avvenimenti. L'interesse dello scrittore non è storico, ma umano. Si potrebbe al limite obiettargli che la nota del paratesto può indurre il lettore in errore. Infatti le informazioni storiche sono poche e imprecise, e chi si avvicina al testo credendo di trovarvi una documentazione esauriente su un episodio marginale e poco conosciuto della Seconda Guerra Mondiale - come suggerito appunto dalla nota del paratesto - non ne coglierà che una qualche lontana reinterpretazione personale, troppo rielaborata per avere un valore storiografico.

Seguendo il ragionamento di Michel Vanoosthuyse, potremmo forse ipotizzare che nel romanzo di Sgorlon c'è un grado debole di relazione con gli avvenimenti del passato. Nel suo saggio sul romanzo storico", Vanoosthuyse spiega che la data, il nome proprio e l'avvenimento storico funzionano come "marqueurs de comparaison». Il lettore è espressamente invitato a rapportarsi agli avvenimenti e alle conoscenze cui il testo si riferisce. Da qui nascono per questo genere le categorie di " fedeltà » e " infedeltà ». In questo senso il romanzo di Sgorlon si situa ad un alto grado di infedeltà storiografica.

Allargando la nostra inchiesta, possiamo allora chiedere : queste caratteristiche sono comuni anche ai romanzi successivi ? Che rapporto hanno questi con la documentazione storiografica?

Anche il secondo romanzo della nostra analisi, La Foiba Grande ${ }^{10}$, è introdotto da una nota paratestuale in cui si specifica che : "Come altri

7. Bruna Sibille-Sizia, La terra impossibile : storia dell'armata cosacca in Friuli, Udine, Doretti, 1991 (1956).

8. Si conta altrimenti un solo altro romanzo sull'argomento, quasi contemporaneo a quello di Sgorlon. Si tratta di Claudio Magris, Illazioni su una sciabola, Roma-Bari, Laterza, 1984, in cui grazie al metodo della ricerca storica si aggira il problema dello scontro e il dolore della guerra è filtrato dalla distanza e dalla pietà del tempo.

9. Michel Vanoosthuyse, Le roman historique : Mann, Brecht, Döblin, Paris, PUF, 1996, p. 60.

10. Carlo Sgorlon, La Foiba Grande, Milano, Mondadori, 1991. 
miei, questo è un libro di invenzione ma anche di storia ». Il romanzo ripercorre un'altra fase drammatica della guerra ed è geograficamente circostanziato ${ }^{11}$. Storicamente, dopo l'8 settembre 1943 il confine ad Est era tutto da definire e l'Italia, che era stato violento invasore in Istria, si trovò in una posizione estremamente delicata. Ci furono episodi di violenza contro fascisti ed italiani (spesso confusi), che si ripeterono in modo più consistente nel maggio 1945, a guerra finita. Le persone venivano giustiziate e gettate nelle foibe, "cavità naturali del terreno ". Il fatto costituisce una delle pagine più tragiche della Seconda Guerra mondiale e la polemica sul numero dei morti è tema costantemente attuale. Nel retro di copertina della prima edizione del settembre 1992 si legge che nelle foibe " vennero gettati, spesso soltanto feriti o ancora vivi, più di diecimila italiani ${ }^{12}$ ".

Qual è la fonte di questo dato ? Gli unici dati « ufficiali » che si hanno, stimati dagli anglo-americani, parlano di duemilaquattrocentosettantadue morti. Sicuramente sono discutibili, perciò aiuta un'ampia consultazione bibliografica. Se un libro militante vuole addirittura dimostrare che le vittime siano solo diciotto, le uniche per cui ci sia stato un processo e dei colpevoli siano stati identificati, la stragrande maggioranza degli studi oscilla intorno ad una stima di quattro/sei mila morti e solo poche voci, sempre molto marcate ideologicamente, propongono una cifra superiore. Nel 2001 una commissione italo-slovena è stata incaricata di fare luce sulla questione e ha parlato di « centinaia di esecuzioni sommarie ». Il dato riportato sul retro di copertina, ripeto, parla di più di 10000 morti.

Il fatto che ci interessa è che la nota non accenna a un dibattito di ampie dimensioni, anzi, prende un dato fazioso, dandolo come assoluto. Quindi, oltre alla scelta forte del soggetto, balza agli occhi la rigida posizione assunta che contribuisce a diffondere nel romanzo una costante fobia antislava e anticomunista, come risulta per esempio dal passo seguente : «V'erano le prove che l'esercito dei boschi, alleato dei comunisti italiani, aveva tentato di prendersi anche il Friuli fino al Tagliamento ${ }^{13}$ ". Anche in questo caso Sgorlon lancia con sicurezza un'accusa che pesa come un macigno : i comunisti italiani fanno combutta con quelli slavi per svendere il Friuli. Solo che, nuovamente, manca il supporto storico. Le rivendicazioni slave su alcuni territori del Friuli sono accertate, ma estenderle fino alla linea del Tagliamento deforma la prova documentaristica in

11. I toponimi sono molto particolareggiati : $c f r$. Umizza, il fiordo di Leme, ecc.

12. La nota è di Bruno Maier.

13. Carlo Sgorlon, op. cit., pp. 226. 
propaganda. La posizione in cui la frase si trova nel testo fa sorgere un dubbio ermenueutico, poiché non si capisce se il dato faccia parte di quella componente di "storia ", cui accenna lo scrittore nel paratesto e che deve offrirci gli elementi della " comparaison", oppure se debba essere interpretata, vista la sua posizione in un'opera di finzione, come dato della diegesi, equiparabile a un personaggio immaginario.

Oltre a questo, la documentazione nel romanzo è scarsa e una costante ambiguità gioca sul doppio livello interpretativo, confondendo la finzione storica con il dato oggettivo e rendendo difficile l'interpretazione. Chi fa l'affermazione sopra citata ? E quali sono i punti di collegamento con il piano «storico » cui si accenna nel paratesto ?

Si tratta di un esempio emblematico, ma questo tipo di dubbio ricorre spesso mentre si legge il romanzo. Il lettore ha due possibilità : dare un valore storiografico a certe affermazioni e quindi avvalorare l'ipotesi del romanzo storico e della combinazione di " fantasia e storia ", oppure considerare queste frasi in un'ottica di finzione senza riferirle in nessun modo ad avvenimenti o a luoghi attinenti alla realtà. Ma la nota paratestuale funziona come una sorta di " patto » con il lettore e lo invita ad una doppia lettura, di finzione e storiografia mescolate. Inoltre la toponomastica e la cronologia circostanziate sembrano avvalorare questa tesi.

Se i riferimenti storiografici paiono dubbi, più chiari sembrano essere quelli letterari. A livello artistico, infatti, il romanzo suggerisce l'ipotesi di un nesso intertestuale con Materada di Fulvio Tomizza, naturale termine di confronto su questo tema. Sgorlon critica lo scrittore italo-istriano perché parla solo delle proprie esperienze personali e perché, pur essendo fortemente interessato all'argomento, non usa mai la parola " foiba " nei suoi romanzi. Eppure la parola « foiba » in Tomizza compare, ed inoltre il tema è toccato, oltre che in Materada, in La quinta stagione ${ }^{14}$ e in L'albero dei $\operatorname{sogni} i^{15}$, a parte il fatto che i problemi etnici sono l'elemento cardine di molte sue opere e in particolare del romanzo Gli sposi di via Rossetti. In Materada, l'attacco mosso al nuovo regime comunista non si articola intorno all'episodio delle foibe, ma si sviluppa in modo più organico attaccando la sostanza culturale della nuova realtà e risulta ideologicamente molto più efficace di quello di Sgorlon.

I romanzi di Sgorlon, più che mirare a un valore storiografico, paiono invece interessarsi al livello ideologico del dibattito. Questa ipotesi sembra

14. Fulvio Tomizza, La quinta stagione, Milano, Mondadori, 1965.

15. Id., L'albero dei sogni, Milano, Mondadori, 1969. 
confermata dall'ultimo dei tre testi, La malga di Sîr ${ }^{16}$, la cui vicenda ripercorre la storia di un episodio particolarmente complesso della resistenza friulana e nazionale. Sgorlon, sapendo di camminare sui carboni ardenti, si tutela scrivendo nel paratesto che "la Malga di Sîr, come altri miei romanzi, è un libro misto di storia e di invenzione. Tutti i personaggi sono frutto di fantasia, ed ogni eventuale contatto tra loro e persone realmente esistite è da considerarsi puramente casuale ». Già nella sua apparente semplicità denotativa la dichiarazione stabilisce un discrimine tra eventi storici e verificabili, e vicende individuali dei personaggi, inventate. Come dire che quanto pertiene alla realtà storica è verità obiettiva e incontrovertibile dei fatti. Ma articolati nella fantasia con personaggi immaginari, anche i fatti obiettivi si caricano del punto di vista del personaggio che li vive, subisce, contrasta o accetta. Gli effetti della finzione non evitano, seguendo un'invisibile frontiera, gli avvenimenti storici, anzi li pervadono. Non c'è discrimine netto tra i due settori mescolati e volti a creare la coerenza romanzesca. Il patto narrativo posto in limine, mentre ribadisce una differenza, sorvola sull'operazione letteraria che viene compiuta nei riguardi del lettore.

Nel romanzo, la vicenda inizia con gli anni del regime e giunge rapidamente al 1943, quando nascono le prime avvisaglie della resistenza. I partigiani titini arrivano nella zona delle Valli del Natisone e si presentano stuprando la giovane protagonista Marianna che per le sue eccezionali doti umane pare in odore di santità. La paura dell'invasione da Est e il dilagare dei comunisti ci accompagna fino al 6 febbraio del 1945 (ibid., p. 264). In quel giorno, nel testo e nella realtà, un centinaio di partigiani garibaldini sale alle malghe vicino al paese di Porzûs e uccide i partigiani di una brigata Osoppo (cattolici repubblicani). La questione solleva ancora oggi polemiche accese ed è inoltre il principale argomento di delegittimazione delle bande garibaldine. Alla base del fatto ci sono accuse reciproche : da un lato i garibaldini avrebbero fatto fronte comune con l'alleato slavo per permettere a Tito di occupare alcune aree del Friuli ; dall'altro i cattolici, in nome di questa paura, avrebbero trattato con i nazisti in funzione anti-comunista, contravvenendo agli ordini del CLN. Nell'imboscata morì Guido Pasolini, il fratello di Pier Paolo, appena arrivato alle malghe col nome di «Ermes ». Si ricordi la poesia dedicata all'episodio, pubblicata sullo Stroligut dell'agosto 1945.

16. Carlo Sgorlon, La malga di Sîr, Milano, Mondadori, 1995. 
Per molti aspetti il romanzo e la ricostruzione storiografica procedono di pari passo. I " rossi " salgono al presidio dicendo che sono sbandati e usando come contatto il partigiano "Dinamite " che andava spesso in malga per recuperare, appunto, esplosivo. Nel romanzo il fatto è mantenuto e il partigiano si chiama "Tritolo ". Uccidono sul luogo i capi e una donna denunciata come spia da Radio Londra e ivi nascosta (in questa sede non è possibile approfondire la questione, piuttosto contorta) e portano gli altri in un bosco in pianura, li processano, risparmiano due sole persone che spergiurano di passare alla formazione garibaldina e uccidono gli altri diciotto. Tutti questi fatti sono fedelmente mantenuti da Sgorlon.

Giovanni Padoan "Vanni ", commissario politico della divisione Garibaldi-Natisone, è uno degli storici più prolifici sull'argomento. In Porzûs ${ }^{17}$ contesta allo scrittore varie imprecisioni, fra cui : la nascita della formazione partigiana Osoppo che Sgorlon mette nelle valli ante tempore ; la strada percorsa dal commando per salire alla malga e il fatto abbastanza sostanziale che il commando stesso non ascese alla malga di nascosto, ma al contrario passando nei paesi e facendosi vedere da numerosi testimoni oculari. Padoan, infine, a proposito di certe descrizioni dei garibaldini, parla di « caricatura indegna».

Mi pare tuttavia che Padoan tralasci alcuni elementi sostanziali. Nel romanzo $\mathrm{i}$ " rossi » sognano di trovare in malga Marianna, la protagonista già stuprata, per poterne abusare sessualmente, ma tutta la storiografia ci assicura che solo il comandante era al corrente della missione e gli altri, ovviamente, ignoravano la presenza di una donna. Merita un'analisi anche l'episodio dei prigionieri fatti scendere a valle con le mani legate dietro la schiena (nel romanzo e nella ricostruzione storiografica). Gli storici si sono sempre interrogati su questa scelta bizzarra : perché far scendere a valle i prigionieri facendosi oltretutto vedere, perché non processarli alla malga ? Sgorlon dà una risposta e fa dire al capo garibaldino "Rasputin » alias " Giacca " che servivano come facchini per razziare i beni del presidio. Ma l'incongruenza logica è evidente : come fanno a fare i facchini con le mani legate dietro la schiena ${ }^{18}$ ? Anche a livello puramente narrativo la soluzione non è convincente.

Il punto nodale è però quello delle violenze agli uccisi in malga. Sgorlon vi si sofferma con dettagli macabri : parla di cranio sfondato a martellate, del seno della donna (la presunta spia) nudo e martoriato da

17. Giovanni Padoan «Vanni ", Porzûs, strumentalizzazione e realtà storica, Monfalcone, Edizioni della Laguna, 2000, p. 167 e segg.

18. Carlo Sgorlon, op. cit., p. 274-278. 
coltellate, di un altro corpo macellato e scannato (ibid., p. 267). Chi li ritrova non aveva " mai visto niente di simile» (ibid., p. 268). Si parla di un medico che salì a constatare " la tortura, il massacro, le coltellate e i colpi di bastone o di mazza » (ibid., p. 269), ma non risulta esserci nessun referto medico. Furono i carabinieri a recuperare le salme.

Nel romanzo, di fatto, i capi comunisti approvano l'esecuzione, ma si scagliano contro Rasputin per le torture, le coltellate, i colpi di mazza che sono " il granello che fa inceppare il meccanismo » (ibid., p. 275). Queste violenze, quindi, assumono un peso fondamentale che sposta l'azione dal livello di scontro armato a quello di ferocia animale. Il lettore si chiede allora se i fatti appertengano al registro della storia o a quello della finzione. Dove passa la linea di demarcazione ostentata nel paratesto ?

Nessun testo storico nomina queste violenze, nemmeno la sentenza 30.4.1954 della Corte d'Assise d'Appello di Firenze sull'eccidio di Porzûs, in cui ci sono tutte le testimonianze dei fatti. Solo Marco Cesselli in "Porzûs, due volti della resistenza », dichiara : "Nacque poi la diceria degli osovani accoltellati, finiti a colpi di martello, e selvaggiamente seviziati. [...] le sevizie non furono mai provate ${ }^{19}$ ". Mi chiedo allora quale sia la fonte di Sgorlon che in un colloquio telefonico del 28 luglio $2003 \mathrm{mi}$ risponde di non ricordare fonti sicure. Si abbandona quindi ad un'esternazione ideologica in cui dice che lui è convinto che siano stati uccisi a colpi di pala e piccone. Per quest'affermazione manca qualsiasi fonte storica e anche la strada della diceria è difficilmente percorribile.

Sgorlon dice di aver letto la sentenza di uno dei processi ${ }^{20}$, ma nella sentenza di Firenze, come detto, non si parla delle violenze. Aggiunge che a lui interessa parlare del Friuli in rapporto agli Slavi, perché

abbiamo corso il rischio di essere posseduti dalla Jugoslavia e dal comunismo e questo è molto grave. Volevo parlare delle efferatezze dei comunisti [...] Gli slavi sono molto nazionalisti e in fondo tribali. In guerra possono arrivare a qualsiasi eccesso. Ho dubbi sul loro ingresso in Europa, devono prima imparare a comportarsi da europei.

Ancora una volta la precisione storiografica latita a favore del fine ideologico.

Ma a livello letterario, e questo interessa in modo primario l'analisi, Sgorlon pratica un capovolgimento dei canoni del romanzo storico. La tradizione e la teoria del genere invitano infatti a citare i personaggi cono-

19. Marco Cesselli, op. cit., p. 98. Questo può essere considerato come il testo più completo sulla vicenda.

20. Sgorlon afferma di aver forse letto gli atti del processo di Lucca. Questa asserzione pare improbabile poiché non sono pubblicati e non sono accessibili. Più probabilmente si riferisce a quelli della sentenza di Firenze, editi. 
sciuti, ma a lasciarli sullo sfondo. Le loro biografie sono spesso troppo dettagliate e conosciute per poterli fare agire liberamente in un romanzo. L'azione deve essere lasciata a figure secondarie. Sgorlon compie un'operazione ibrida : rende protagonisti della storia i personaggi celebrati dalla storiografia, dando loro una vita complessa e inventata nella finzione, ma mantenendo un forte aggancio con il corrispettivo sul piano storiografico (l'esempio di Dinamite-Tritolo è rivelatore). I personaggi, però, sono presentati come pura creazione letteraria, senza alcun " contatto tra loro e persone realmente esistite ». Quindi, formalmente, il «Tritolo» della finzione non rinvia in nessun modo al "Dinamite " della resistenza, pur compiendo la stessa funzione nell'azione.

Qual è, allora, la componente storica del libro che è presentato come un " misto di storia e di invenzione " ? Le convenzioni del genere sono stravolte. I personaggi storici non sono citati per restare sullo sfondo, ma sono sostituiti da altri, frutto della finzione, che alludono fortemente a loro. Questi doppi quasi "platonici », queste copie delle copie, ripercorrono le gesta di un episodio storico ben determinato, rielaborandolo liberamente. Nel romanzo storico l'invenzione è solitamente riservata al vuoto lasciato dalla storiografia, Sgorlon invece pone l'invenzione a sostituzione dei dati storiografici ${ }^{21}$.

La malga di Sîr si può considerare il primo vero romanzo centrato sulla spinosa questione di Porzûs. Altri testi di finzione hanno affrontato il difficile tema solo in modo marginale, ma sono stati più rispettosi delle regole del genere : Claudio Magris in Illazioni su una sciabola accenna solo vagamente alla vicenda parlando di " tradimento fratricida » e di " innominabile strage di Porzûs ». Il romanzo resistenziale $I l$ Ghebo ${ }^{22}$ di Elio Bartolini, libro che comunque, per l'antiortodossa ricostruzione della resistenza, ebbe una travagliata storia editoriale e, sebbene scritto nel 1946, fu pubblicato solo nel 1970, lascia la vicenda nel vago, facendola arrivare in modo confuso alle orecchie di un gruppo di partigiani braccati, concentrando invece l'attenzione del romanzo sulla difficoltà di creare un comando unico tra fazzoletti rossi e verdi.

Non sono il solo a contestare a Sgorlon l'abitudine all'imprecisione storica. Nel libro di Liana Nissim, Sgorlon teste insolente (1985), in un confronto con alcuni critici, Sgorlon, attaccato ripetutamente (a proposito di altri romanzi) proprio sul suo modo di documentarsi, risponde alla

21. Inoltre Sgorlon tocca un argomento delicatissimo e lo fa con il tatto di un elefante, aggiungendo violenza ideologica a una questione ancora non risolta.

22. Elio Bartolini, Il Ghebo, Muggia, Editrice Santi Quaranta, 2000 (1946). 
fine che lui è uno scrittore impaziente, non uno scrittore filologo e che, tutto sommato, ciò non gli dispiace molto poiché lui raccoglie quello che ha dentro ${ }^{23}$. A questa nota intimista si potrebbe replicare che l'alterazione dei fatti ha un preciso fine ideologico e non è solo ingenua pigrizia documentaristica. Così per esempio, una caratteristica de La malga di Sîr è la rappresentazione diretta e forte della violenza, mentre, al contrario, di solito, la violenza è poco presente, gli eventi bellici sono sempre marginali, lontani, incomprensibili alla semplicità del popolo. Di fronte a questo criterio narrativo le efferratezze de La malga di Sîr costituiscono uno scarto, non privo di valenze ideologiche. Altrove la guerra è spiegata come una malattia, un virus che passa, di cui non si conoscono le cause.

Di solito, la violenza descritta nei romanzi di Sgorlon è quella privata, soprattutto quella subita dalle donne. Nei suoi romanzi si ripetono con morbosa insistenza le scene di stupro, anche queste determinate in certo senso dalla fatalità, come necessario corollario della guerra. Ma la loro dinamica è fiabesca (tra l'altro è interessante verificarne il sostanziale rispetto delle funzioni fondamentali della favola delineate da Propp) ed è palesata in un passo de La Foiba Grande in cui Vera, la giovane e fresca protagonista che ha subito l'affronto, raccontando storie di orchi e lupi si rende conto che quelle cose esistono per davvero.

L'aspetto favolistico dei romanzi è suggerito da diversi elementi testuali, come l'improbabile antimimetismo dei dialoghi che riconducono ad un referente mitico leggendario più che ad una situazione reale : giovani della campagna con una lingua da maestrini, soldati tedeschi che parlano con il bon ton di un'educanda, valorosi cosacchi dal gentile idioletto allegorico ci confermano che le intenzioni dell'autore non sono quelle di rappresentare la realtà, ma di offrire una sua visione del mondo.

Quello di Sgorlon è un mondo arcaico e contadino, svelato anche dalla patina linguistica. Prendendo in prestito un'espressione propria alla fotografia, diremo che Sgorlon, come in un viraggio seppia, ci regala scenari vecchi, tradizionali e regionali, nei riferimenti culturali, nelle metafore, negli usi semantici e sintattici, e fa uso di un linguaggio di afona correttezza monocorde che ambisce ad una nitida forma scolastica in cui le forme dialettali "pure » sono inserti linguistici abbastanza occasionali e non intervengono a modificare la sintassi. Uno stile per il quale si potrebbe rievocare lo stile neo-antico di cui parla Mario Perniola ${ }^{24}$, o una citazione di John Barth :

23. Liana Nissim, Sgorlon teste insolente: materiali per un'ermeneutica e una dichiarazione poetica di Carlo Sgorlon, Udine, I quadeni del Gamajun, 1985, p. 133.

24. Mario Perniola, Il pensiero neo-antico, tecniche e possessione nell'arte e nel sapere del mondo contemporaneo, Milano, Associazione culturale mimesis, 1995. 
Un buon numero di romanzieri contemporanei scrive romanzi da inizio secolo, anche se con un linguaggio più o meno da metà secolo e con personaggi e temi contemporanei : ciò li rende (per me) assai meno interessanti di quegli eccellenti autori che sono contemporanei anche tecnicamente : Joyce e Kafka, per esempio, nella loro epoca, e nella nostra, Samuel Beckett e Jorge Luis Borges ; [a questa categoria] attribuirei tutti quei romanzieri che, chi peggio chi meglio, scrivono non come se il XX secolo non esistesse, ma come se i grandi scrittori degli ultimi sessant'anni non fossero mai esistiti ${ }^{25}$.

Questi elementi contribuiscono a rendere vivo quel mondo antico e probo che ci proteggerebbe da strane idee politiche, dai cambiamenti e dai popoli cattivi e che, come sottolinea anche Maier, è mitico, inventato, un misto di ricordi edulcorati e di influenza della letteratura fantastica latinoamericana, una delle fonti principali riconosciute allo scrittore. Per questa rappresentazione convenzionale del Friuli troverei inoltre delle radici in quella poetica della " polenta » di cui ha parlato Pasolini nello Stroligut a proposito della tradizione letteraria regionale in friulano, e in questo senso mi pare che la critica abbia fortemente latitato.

Per Sgorlon la letteratura è chimera e fantasia proprio grazie a una imprecisa conoscenza dei fatti storici. Come dice lo scrittore stesso : « se lei legge Sgorlon solo per trovare delle testimonianze, fa bene a cambiare autore ${ }^{26}$ ". Ma dietro questo sottile discrimine si nasconde, a mio avviso, la fessura in cui infiltrare un'ideologia. Mi sembra che Sgorlon, alterando le abitudini del romanzo storico, tenda piuttosto a darci un romanzo " ideologico ", il cui primo fine pare quello di convincere della propria posizione riguardo ad eventi poco conosciuti, i quali, proprio perché verificabili sul piano storiografico, inducono nel lettore una forma di acquiescenza, un'accettazione passiva di quanto raccontato grazie anche alla dichiarazione liminare. La verifica storica mostra al contrario come la posizione di Sgorlon si contrapponga, per certi aspetti, ai giudizi canonici della storiografia. I valori umani attribuiti con profusione allo scrittore appaiono spesso secondari rispetto al suo fine polemico. La sua opera, prima che opera di comprensione, pietà e simpatia per le vittime, vuole essere un baluardo eretto a difesa della civiltà cristiana e liberale - come dice lo stesso autore - ma in un atteggiamento di sconcertante disinvoltura verso i fatti storici.

* Une version légèrement différente du même article a été publiée en 2004 dans la revue Metodi \& Ricerche, sans aucune mention du Colloque de Grenoble.

25. John Barth, La letteratura dell'esaurimento, in P. Carraveta, P. Spedicato, Postmoderno e letteratura, Milano, Bompiani, 1984, pp. 49-60, pp. 50-51.

26. Liana Nissim, Sgorlon teste insolente : materiali per un'ermeneutica e una dichiarazione poetica di Carlo Sgorlon, Udine, I quaderni del Gamajun, 1985, p. 132. 
Bibliografia consultata (PER ARgomenti e Per data)

\section{Storiografia}

\section{Sui cosacchi in Friuli}

Gortani M., Il martirio della Carnia, Udine, Leonardo editrice, 2000 (relazione 30 maggio 1945).

Ermacora C., La patria era sui monti, Udine, La panarie, 1945.

Montanelli I., L'Italia della guerra civile, Milano, Rizzoli, 1985.

Carnier P.A., L'armata cosacca in Italia 1944-1945, Milano, Mursia, 1990.

Pansa G., Il gladio e l'alloro : l'esercito di Salò, Milano, Mondatori, 1991.

Zanier L., Carnia Kosakenland Kazackaja Zemlja : storiutas di fruts ta guera, racconti di ragazzi in guerra, prefazione di Mario Rigoni Stern, Udine, Edizioni Mittelcultura, 1995.

Sul fenomeno delle « foibe "

Pacor M., Confine orientale. Questione nazionale e Resistenza nel Friuli Venezia Giulia, Milano, Feltrinelli, 1964.

Miccoli G., Risiera e Foibe. Un accostamento aberrante, in "Bollettino dell'istituto regionale per la storia del movimento di liberazione nel Friuli-Venezia Giulia ", a. IV n. 1, aprile 1976.

Dallo squadrismo fascista alle stragi della risiera : Trieste-IstriaFriuli-1919-1945, Trieste, Aned, 1974.

Cox G., La corsa per Trieste, Gorizia, Editrice Goriziana, 1985.

Spazzali R., Foibe, un dibattito ancora aperto, Trieste, Editrice lega nazionale, 1990.

Pirina M., d'Antonio A., Adria Storia, Pordenone, Centro studi e ricerche storiche "Silentes loquimur », 1993.

Albo d'oro. La Venezia Giulia e la Dalmazia nell'ultimo conflito mondiale, Trieste, Ed. Unione degli istriani, 1994.

Spazzali R., Campi di sterminio e foibe : silenzi ed omissioni, in "Udine, Scuola cattolica di cultura", n. 2, 1996.

Foibe. Il peso del passato, a cura di Giampaolo Valdevit, Venezia, Marsilio, 1997.

Pupo R. , Le foibe giuliane 1943-1945, in Friuli e Venezia Giulia : storia del '900, Gorizia, LEG, 1997, p. 389-400.

Cernigoi C., Operazione Foibe a Trieste, Udine, Kappa Vu, 1997.

Venezia Giulia 1943-1945, a cura di A. Nuvoli, [S.1.], Comitato regionale dell'ANPI del FVG, 1998. 
Foibe e deportazioni, per ristabilire la verità storica, a cura di Alberto Buvoli, Udine, "Quaderni della resistenza », 1998.

Pupo R. , Guerra e dopoguerra al confine orientale d'Italia (1938-1956), Udine, Del Bianco, 1999.

Oliva G., La resa dei conti : aprile-maggio 1945: foibe, piazzale Loreto e giustizia partigiana, Milano, Mondatori, 1999.

Rumici G., Infoibati 1943-1945 : i nomi, i luoghi, i testimoni, i documenti, Milano, Mursia, 2002.

Oliva G., Foibe, Le stragi negate degli italiani della Venezia Giulia e dell'Istria, Milano, Mondadori, 2002.

\section{Sull'eccidio di Porzûs}

Colonnello G.A., Guerra di liberazione : Friuli Venezia Giulia, zone jugoslave, Udine, Edizioni Friuli, 1965.

Padoan G. "Vanni », Abbiamo lottato insieme. Partigiani italiani e sloveni al confine orientale, Udine, Del Bianco, 1966.

Bocca G., Storia dell'Italia partigiana, Bari, Laterza, 1966, p. 477.

Cresta P., Un partigiano dell'Osoppo al confine orientale, Udine, Del Bianco, 1969.

Pasolini P.P., Sulla morte di Guido, in "Vie nuove », 15 settembre 1971.

Cesselli M., Porzûs due volti della resistenza, Milano, La Pietra, 1975.

Mascialino R., La resistenza dei cattolici in Friuli, 1943-1945, Udine, La nuova base, 1978.

Gervasutti S., La stagione della Osoppo, Udine, La nuova base, 1981.

Per rompere un silenzio più triste della morte : testo della sentenza 30.4.1954 della Corte d'Assise d'Appello di Firenze sull'eccidio di Porzus con prefazione di Gianfranco Bianchi e note di Silvano Silvani, Udine, La Nuova base, 1983.

Padoan G. "Vanni ", Un'epopea partigiana alla frontiera tra due monti, Udine, Del Bianco, 1984.

Kersevan A., Porzûs, Dialoghi sopra un processo da rifare, Udine, Edizioni Kappa Vu, 1995.

Franceschini D., Porzûs. La resistenza lacerata, Trieste, Isrts Trieste, 1996.

Gervasutti S., Il giorno nero di Porzûs, Venezia, Marsilio, 1997.

Lenoci A., Porzûs, la resistenza tradita, Bari, Laterza, 1998.

Padoan G. "Vanni ", Porzûs, strumentalizzazione e realtà storica, Mariano del Friuli, Monfalcone, Edizioni della laguna, 2000.

Buvoli A., L'eccidio di Porzûs : ipotesi interpretative, in "Storia contemporanea in Friuli », Udine, Istituto friulano per la storia del movimento di liberazione, anno XXXI-2001, n. 32, pp. 9-24. 


\section{Critica letteraria}

Sgorlon C., La narrativa friulana nel dopoguerra, in " Majano nuova ", numero dedicato alla arti e alle lettere, Majano, 1970, p. 20-25.

Guagnini E., La narrativa italiana in Friuli tra i 1968 e il 1969, Udine, "Ce fastu? », n. 44-47, 1968-71, pp. 147-157.

- Simbolismo, immaginazione e realtà in Carlo Sgorlon narratore, "Ce fastu ?", n. 48-49, 1973, pp. 118-141.

De Lorenzi A., Capire Sgorlon, Udine, «La Panarie », 4/1973, pp. 33-36.

Il poema dell'anima friulana, Udine, " Messaggero Veneto ", 8-5-1977.

Guagnini E., Narrativa italiana in Friuli tra il 1976 e il 1977, in in Udine, "Ce fastu? ", 1977, p. 177-193.

Giuliani A., Cantastorie friulano tra miti e fantasmi, in "La repubblica ", 9/4/1977.

De Lorenzi A., Gli dei torneranno, Udine, «La Panarie », giugno 1977.

Passamonte O., Il magico e il reale in Carlo Sgorlon, tesi di laurea [S.1.], relatore Giuseppe Amoroso, 1978.

Maier B., Carlo Sgorlon, Firenze, La Nuova Italia, 1984.

BarthJ., La letteratura dell'esaurimento, in P Carraveta, P. Spedicato, Postmoderno e letteratura, Milano, Bompiani, 1984, pp. 49-60.

NissimL., Sgorlon teste insolente : materiali per un'ermeneutica e una dichiarazione poetica di Carlo Sgorlon, Udine, I quadeni del Gamajun, 1985.

D'Aronco G., Le illusioni perdute di un erede, Udine, "Ce fastu ? ", LXII, 1986, pp.342-345.

De Lorenzi A., Sgorlon C., Presentazione de La Foiba Grande, Biblioteca Comunale V. Joppi, Udine, 1993.

Toscani C., Invito alla lettura di Sgorlon, Milano, Mursia, 1994.

\section{Sul romanzo storico}

Manzoni A., Del romanzo storico e, in genere, décomponimenti misti di storia e d'invenzione, in Edizione nazionale ed europea delle opere di Alessando Manzoni, Milano, Centro Nazionale di Studi Manzoniani, 2000, vol. XIV.

Lukács G., Le roman historique, Paris, Editions Payot, 2000 (1937).

Il romanzo storico, a cura di L. Lattarulo, Roma, Editori Riuniti, 1978.

Singler C., Le roman historique contemporain en Amérique Latine. Entre mythe et ironie, Paris, L'Harmattan, 1993.

Vanoosthuyse M., Le roman historique : Mann, Brecht, Döblin, Paris, PUF, 1996. 
Le roman historique. Récit et histoire, sous la direction de Dominique Peyrache-Leborgne et D. Couégnas, Nantes, Éditions Pleins Feux, 2000.

\section{Poesie e romanzi}

Pasolini P.P., Corus in morte di Guido, in Stroligut n.1, agosto 1945, San Vit, Stamparia Primon, p. 3-4.

Sibille-Sizia B., La terra impossibile : storia dell'armata cosacca in Friuli, Udine, Doretti, 1991 (1956).

—, Avinis, paese senza peccato, Udine, Doretti, 1960.

Tomizza F., Materada, Milano, Mondadori, 1960.

—, La quinta stagione, ibid., 1965.

—, L'albero dei sogni, ibid., 1969.

Magris C., Illazioni su una sciabola, Cariplo Laterza, 1984.

Tomizza F., Gli sposi di via Rossetti, Milano, Mondadori, 1986.

Sibille-Sizia B., Il fronte di fango, Udine, Doretti, 1988.

Revelli N., Il disperso di Marburg, Torino, Einaudi, 1994. 